\title{
New universal relations for nonlinear isotropic elastic materials
}

\author{
K.R. RAJAGOPAL ${ }^{(1)}$ and ALAN S. WINEMAN (2) \\ (1) Department of Mechanical Engineering, University of Pittsburgh, Pittsburgh, Pennsylvania 15260, U.S.A. \\ (2) Department of Mechanical Engineering and Applied Mechanics, The University of Michigan, Ann Arbor, \\ Michigan 48109, U.S.A.
}

Received 26 June 1985; in revised form 11 September 1985

\begin{abstract}
A nonlinear isotropic elastic block is subjected to a homogeneous deformation consisting of simple shear superposed on triaxial extension. Two new relations are established for this deformation which are valid for all nonlinear elastic isotropic materials, and hence are universal relations. The first is a relation between the stretch ratios in the plane of shear and the amount of shear when the deformation is supported only by shear tractions. The second relation is established for a thin-walled cylinder under combined extension, inflation and torsion. Each material element of the cylinder undergoes the same local homogeneous deformation of shear superposed on triaxial extension. The properties of this deformation are used to establish a relation between pressure, twisting moment, angle of twist and current dimensions when no axial force is applied to the cylinder. It is shown that these relations also apply for a mixture of a nonlinear isotropic solid and a fluid.
\end{abstract}

\section{Introduction}

It is well known that unequal normal tractions are required if a homogeneous simple shear deformation is to be produced in a nonlinear elastic isotropic solid. It can be inferred that if shear tractions are applied to a block of such material, but normal tractions are not, then its faces will draw together or spread apart by an amount which depends on the amount of shear deformation [1]. This is known as the POYNTING effect.

In the present work, we derive a universal relation associated with the changes in the dimensions of the block in virtue of this effect. The block is assumed to be in a state of homogeneous deformation consisting of a simple shear superposed on triaxial extension. The shear is given. The stretch ratios which determine the spacing between the faces of the block are found from the condition that the normal tractions vanish. An analysis of the resulting equations leads to the major result of this work, a new 'universal relation'. In particular, we establish that when the normal tractions vanish, there exists a relation between the stretch ratios in the shear plane and the amount of shear which does not depend on the form of the strain energy density function. Furthermore, this relation holds for both incompressible and compressible materials.

We discuss next, the application of the previous results to the problem of the combined extension, twisting and inflation of a thin-walled cylinder. This is possible since each material element in a thin-walled cylinder undergoes the same simple shear 
superposed on triaxial extension. In this case, it is shown that there is a universal torque-twist relation when the axial force vanishes and the radius, length and inflating pressure are held fixed.

Next, we proceed to discuss the problem of the shearing of a block of material, within the context of the theory of a mixture of an isotropic elastic solid infused with a fluid, the mixture being subject to the same conditions as the purely elastic solid. We show that a universal relation once again holds and is independent of the fluid content of the mixture. It is easy to conclude from this analysis that a universal relation can be established in the case of twisting and inflation of a thin-walled cylindrical mixture.

A discussion of the basic deformation is presented in Section 2. In Section 3, expressions for the tractions on a block of a nonlinear elastic isotropic solid are established and the first universal relation is derived. Results for the combined extension, inflation and torsion of a thin-walled circular cylinder are developed in Section 4. Finally the results of Sections 3 and 4 are extended, in Section 5, to a mixture of an isotropic elastic solid and a fluid.

\section{Simple shear superposed on triaxial extension}

Consider a body whose configuration in the reference state is a block. With respect to a Cartesian coordinate system, let $X_{i}$ denote the coordinates of a particle in the reference state and $x_{i}$ denote its coordinates in a deformed state. Let the block be subjected to the homogeneous deformation

$$
\begin{aligned}
& x_{1}=\lambda_{1} X_{1}+\kappa \lambda_{2} X_{2}, \\
& x_{2}=\lambda_{2} X_{2}, \\
& x_{3}=\lambda_{3} X_{3} .
\end{aligned}
$$

In this deformation, the block undergoes changes in length along each coordinate axis, followed by a simple shear in which planes $x_{2}=$ constant displace along the $x_{1}$ direction. Parameter $\kappa$ represents the displacement along the $x_{1}$ axis per unit current length in the $x_{2}$ direction.

The deformation gradient $F$, the Cauchy-Green tensor $B=F F^{\mathrm{T}}$ and $B^{2}$ are given by:

$$
\begin{aligned}
& \boldsymbol{F}=\left[\begin{array}{ccc}
\lambda_{1} & \kappa \lambda_{2} & 0 \\
0 & \lambda_{2} & 0 \\
0 & 0 & \lambda_{3}
\end{array}\right], \\
& \boldsymbol{B}=\left[\begin{array}{ccc}
\lambda_{1}^{2}+\kappa^{2} \lambda_{2}^{2} & \kappa \lambda_{2}^{2} & 0 \\
\kappa \lambda_{2}^{2} & \lambda_{2}^{2} & 0 \\
0 & 0 & \lambda_{3}^{2}
\end{array}\right], \\
& \boldsymbol{B}^{2}=\left[\begin{array}{ccc}
\left(\lambda_{1}^{2}+\kappa^{2} \lambda_{2}^{2}\right)^{2}+\kappa^{2} \lambda_{2}^{4} & \kappa \lambda_{2}^{2}\left[\lambda_{2}^{2}+\lambda_{1}^{2}+\kappa^{2} \lambda_{2}^{2}\right] & 0 \\
\kappa \lambda_{2}^{2}\left[\lambda_{2}^{2}+\lambda_{1}^{2}+\kappa^{2} \lambda_{2}^{2}\right] & \lambda_{2}^{4}+\kappa^{2} \lambda_{2}^{4} & 0 \\
0 & 0 & \lambda_{3}^{4}
\end{array}\right] .
\end{aligned}
$$


The principal invanants of $B$ are given by:

$$
\begin{aligned}
& I_{1}=\lambda_{1}^{2}+\lambda_{2}^{2}+\lambda_{3}^{2}+\kappa^{2} \lambda_{2}^{2}, \\
& I_{2}=I_{3}\left(\frac{1}{\lambda_{1}^{2}}+\frac{1}{\lambda_{2}^{2}}+\frac{1}{\lambda_{3}^{2}}+\frac{\kappa^{2}}{\lambda_{1}^{2}}\right), \\
& I_{3}=\left(\lambda_{1} \lambda_{2} \lambda_{3}\right)^{2} .
\end{aligned}
$$

Let the block in its reference state be bounded by the pairs of surfaces $X_{i}= \pm X_{i}^{0}$. Consider the surface $X_{1}=X_{1}^{0}$. Its equation in the deformed state is

$$
\frac{x_{1}-\kappa x_{2}}{\lambda_{1}}=X_{1}^{0} \text {. }
$$

The normal traction $N_{1}$ and the shear traction $L$ on this surface are expressed in terms of the components of the Cauchy stress tensor with respect to the Cartesian coordinate system by the relations

$$
\begin{aligned}
& N_{1}=\frac{\sigma_{11}+\kappa^{2} \sigma_{22}-2 \sigma_{12} \kappa}{1+\kappa^{2}}, \\
& L=\frac{\kappa\left(\sigma_{11}-\sigma_{22}\right)+\sigma_{12}\left(1-\kappa^{2}\right)}{1+\kappa^{2}} .
\end{aligned}
$$

\section{Isotropic elastic solids}

Let $W$ denote the strain energy density per unit volume in the reference state, and $\rho_{0}$ and $\rho$ the mass densities in the reference and current states, respectively. Let $W_{\alpha}=$ $\partial W / \partial I_{\alpha}$. The constitutive equations for compressible, and incompressible, isotropic elastic solids are written, respectively, in the form

$$
\begin{aligned}
& \sigma=2 \frac{\rho}{\rho_{0}} I_{3} W_{3} I+\frac{\rho}{\rho_{0}} \hat{\sigma}, \\
& \sigma=-p I+\hat{\sigma},
\end{aligned}
$$

where

$$
\hat{\sigma}=2\left(W_{1}+I_{1} W_{2}\right) B-2 W_{2} B^{2} .
$$

Since the deformation is homogeneous (as is the material), the equations of equilibrium are automatically satisfied (with $p$ a constant in the incompressible case).

Wineman and Gandhi [2] established the following universal relation for simple shear superposed on triaxial extension of isotropic elastic solids,

$$
\sigma_{11}-\sigma_{22}=\left(\frac{\lambda_{1}^{2}+\lambda_{2}^{2} \kappa^{2}-\lambda_{2}^{2}}{\kappa \lambda_{2}^{2}}\right) \sigma_{12} \text {. }
$$

It is easily verified by direct substitution from (2.3) and (2.4) into (3.3) that

$$
\hat{\sigma}_{11}-\hat{\sigma}_{22}=\left(\frac{\lambda_{1}^{2}+\lambda_{2}^{2} \kappa^{2}-\lambda_{2}^{2}}{\kappa \lambda_{2}^{2}}\right) \hat{\sigma}_{12} \text {. }
$$


Note that relation (3.4) can be obtained from (3.5) for any expression of the form

$$
\boldsymbol{\sigma}=\boldsymbol{P I}+A \hat{\boldsymbol{\sigma}} \text {. }
$$

Hence, (3.4) holds for both compressible and incompressible materials.

By using the universal relation (3.4), the shear traction $L$ on the surface $X_{1}=X_{1}^{0}$ in the deformed state can be written as

$$
L=\frac{\lambda_{1}^{2}}{\lambda_{2}^{2}} \frac{\sigma_{12}}{1+\kappa^{2}} .
$$

The normal traction $N_{1}$ on this surface can be written as

$$
N_{1}=\frac{A\left(\lambda_{1}, \lambda_{2}, \kappa\right) \sigma_{11}+B\left(\lambda_{1}, \lambda_{2}, \kappa\right) \sigma_{22}}{1+\kappa^{2}},
$$

where

$$
A\left(\lambda_{1}, \lambda_{2}, \kappa\right)=\frac{\lambda_{1}^{2}-\kappa^{2} \lambda_{2}^{2}-\lambda_{2}^{2}}{\lambda_{1}^{2}+\left(\kappa^{2}-1\right) \lambda_{2}^{2}}
$$

and

$$
B\left(\lambda_{1}, \lambda_{2}, \kappa\right)=\frac{\kappa^{2}\left[\lambda_{1}^{2}+\left(\kappa^{2}-1\right) \lambda_{2}^{2}+2 \lambda_{2}^{2}\right]}{\lambda_{1}^{2}+\left(\kappa^{2}-1\right) \lambda_{2}^{2}} .
$$

Suppose the surface $X_{1}=X_{1}^{0}$ is to be free of shear traction. Then $L=0$, which implies by (3.7) that $\sigma_{12}=0$. It can be shown from (2.3)-(2.5) and (3.1)-(3.3) that

$$
\sigma_{12}=C\left[W_{1}+\lambda_{3}^{2} W_{2}\right] \kappa \lambda_{2}^{2},
$$

where $C=1$ for an incompressible material and $C=\rho / \rho_{0}$ for a compressible material. Since we expect that the shear strain and the shear stress have the same sign, we require that $W_{1}+\lambda_{3}^{2} W_{2}>0$. Then $L=0$ if and only if $\kappa=0$, in which case there is no shear deformation. In the following discussion let $\kappa \neq 0$ be specified.

Now, let the normal tractions vanish on the surface $X_{1}=X_{1}^{0}$ and $X_{2}=X_{2}^{0}$. Since $X_{2}=X_{2}^{0} \Rightarrow x_{2}=\lambda_{2} X_{2}^{0}=$ constant, the only normal stress acting on this surface is $\sigma_{22}$. Thus if the normal traction $\mathrm{N}_{2}$ on $x_{2}=$ constant is to be zero, then $\sigma_{22}=0$. It is to be noted at this juncture that we make no assumptions regarding the stresses acting on the faces $x_{3}= \pm \lambda_{3} X_{3}$. Thus, we are not requiring here, for instance that $\sigma_{33}=0$. Consider first the consequences of the condition that $\sigma_{22}=0$. By (3.4), (3.7) and (3.8), $N_{1}$ can be related to the shear traction by

$$
N_{1}=\left(\lambda_{1}^{2}-\lambda_{2}^{2}-\kappa^{2} \lambda_{2}^{2}\right) L / \kappa \lambda_{1}^{2} \text {. }
$$

Finally, consider the vanishing of the normal stress $N_{1}$ on the slanted surface formed by $X_{1}=X_{1}^{0}$. Since $L \neq 0$, it follows that $N_{1}=0$ if and only if

$$
\lambda_{1}^{2}=\lambda_{2}^{2}\left(1+\kappa^{2}\right) \text {. }
$$

This is the result to be established.

\section{Comment 1}

The relation between $\lambda_{1}$ and $\lambda_{2}$ in (3.13) depends only on $\kappa$. Since it is independent of any particular choice of $W$, it is valid for all isotropic materials. Relation (3.13) is a new universal relation. 

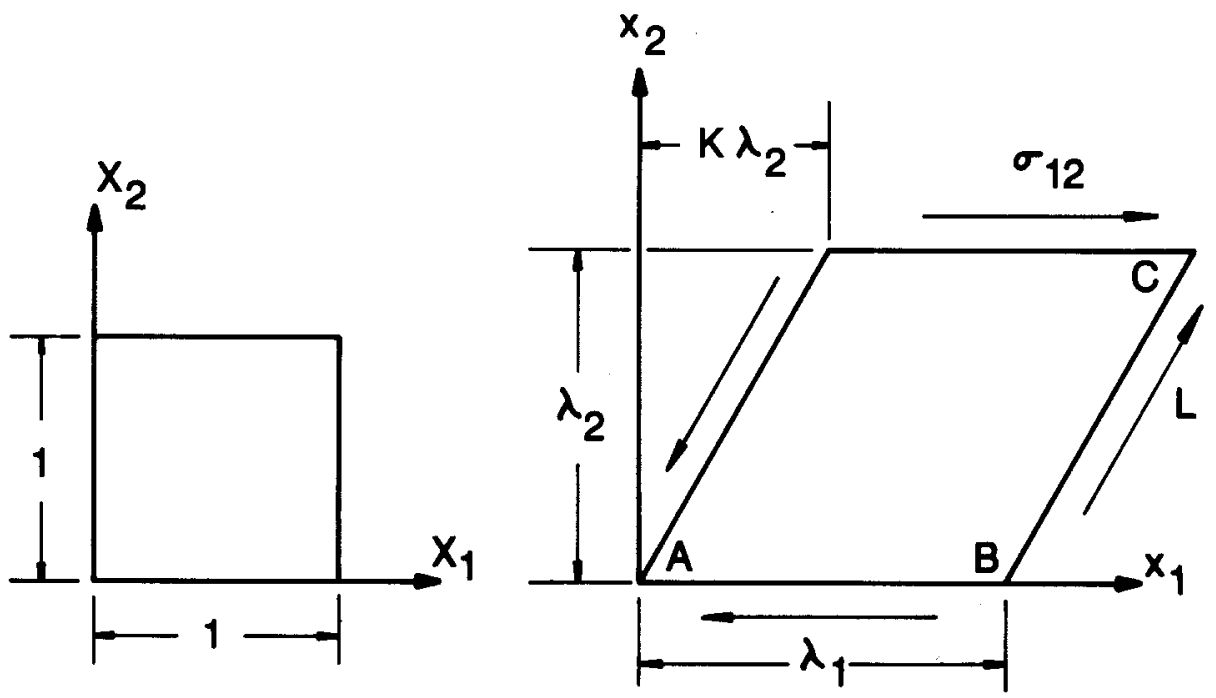

Figure 1. Deformed state of unit block when $\sigma_{12}=N_{1}=0$

\section{Comment 2}

Relation (3.13) holds for both incompressible and compressible isotropic elastic materials.

\section{Comment 3}

Relation (3.13) is independent of the normal stress on surfaces $X_{3}= \pm X_{3}^{0}$.

\section{Comment 4}

Suppose $\sigma_{33}=0$. If the material is compressible, then three equations for $\lambda_{1}, \lambda_{2}, \lambda_{3}$ are given by (3.13) and the two equations obtained by setting $\sigma_{22}=\sigma_{33}=0$ in (3.1).

If the material is incompressible, then $I_{3}=1$ and (2.5) gives $\lambda_{1} \lambda_{2} \lambda_{3}=1$, so that there are only two independent stretch ratios. Three equations for the two independent stretch ratios, say $\lambda_{1}$ and $\lambda_{2}$, and the indeterminate scalar $p$ in (3.2) are provided by (3.13) and the two equations obtained by setting $\sigma_{22}=\sigma_{33}=0$ in (3.2). For a NeoHookean material, for which $W_{2}=0$, and $W_{1}$ is a constant, in (3.3) it is found that

$$
\begin{aligned}
& \lambda_{1}=\left(1+\kappa^{2}\right)^{1 / 3}>1, \\
& \lambda_{2}=\lambda_{3}=\left(1+\kappa^{2}\right)^{-1 / 6}<1 .
\end{aligned}
$$

\section{Comment 5}

Relation (3.13) has an interesting geometric interpretation. As seen in Figure 1, when no normal tractions are applied, $\overline{A B}=\overline{B C}$.

\section{Extension, twisting and inflation of a thin-walled circular cylinder}

Consider a thin-walled circular cylinder which is subjected to axial force $F$, twisting moment $T$ and pressure $p$ on its inner surface. As discussed in [2], the deformation of the cylinder is such that each material element undergoes the local homogeneous deformation described in Section 2. Thus, this problem represents a convenient experimental configuration for studying the POYNTING effect. 
In its reference state the cylinder has length $L_{0}$, mean radius $R_{0}$ and wall thickness $h_{0}$. In its deformed state it has length $L$, mean radius $R$ and wall thickness $h$. The $X_{1}$, $X_{2}, X_{3}$ axes of the homogeneous deformation of Section 2 correspond, locally, to the $\theta, Z, R$ directions on the cylinder. By using the assumption that the cylinder is thin walled, i.e. $h_{0} / R_{0} \ll 1$, variation of quantities through the cylinder wall can be neglected, and all material elements can be regarded as undergoing the same homogeneous deformation. The parameters in (2.1) are then given by

$$
\begin{array}{ll}
\lambda_{1}=R / R_{0}, & \lambda_{3}=h / h_{0}, \\
\lambda_{2}=L / L_{0}, & \kappa=\frac{R \psi}{L}=\frac{R \psi}{\lambda_{2} L_{0}},
\end{array}
$$

where $\psi$ is the angle of rotation of one end of the cylinder with respect to the other. The relations between $F, T, p$ and the stress components are given by

$$
\begin{aligned}
& F=\sigma_{22} 2 \pi R h, \\
& T=\sigma_{12} 2 \pi R^{2} h, \\
& p=\sigma_{11} h / R .
\end{aligned}
$$

We now consider the implication of the condition that each material element of the cylinder be in the state which was discussed in Section 3. First, let $\sigma_{22}=0$, which by (4.2) ${ }_{1}$ corresponds to the condition that the axial force $F=0$. Then the local universal relation * (3.4) reduces to

$$
\sigma_{11}=\left(\frac{\lambda_{1}^{2}+\lambda_{2}^{2} \kappa^{2}-\lambda_{2}^{2}}{\kappa \lambda_{2}^{2}}\right) \sigma_{12}
$$

By (4.1) and (4.2), this can be expressed as the global universal relation given by

$$
\frac{p}{T}=\frac{\left(\frac{R_{0}}{R}\right)^{4}\left(\frac{L}{L_{0}}\right)\left\{\left(\frac{R}{L}\right)\left(\frac{L_{0}}{R_{0}}\right)^{2}\left[1+\left(\frac{R_{0}}{L_{0}}\right)^{2} \psi^{2}\right]-1\right\}}{2 \pi R_{0}^{4} L_{0} \psi}
$$

This is a new universal relation and was overlooked in [2]. Note that it is valid for any value of the twist angle $\psi$.

Before deformation, the surface $X_{1}=X_{1}^{0}$ in Section 2 is vertical and corresponds to an internal surface in the cylinder which is bounded by generator lines on the inner and outer cylindrical surfaces and radial line segments. After deformation, the surface $X_{1}=X_{1}^{0}$ is slanted and corresponds to a helical internal surface bounded by the helices formed by the generators and the radial lines. By (3.7) and (3.12) and (4.2) ${ }_{2}$ the normal stress on this surface can be related to the twisting moment by

$$
N=\frac{\left(\lambda_{1}^{2}-\lambda_{2}^{2}-\kappa_{2}^{2} \lambda_{2}^{2}\right)}{\kappa \lambda_{1}^{2} \lambda_{2}^{2} \lambda_{3}\left(1+\kappa^{2}\right)} \frac{T}{2 \pi R_{0}^{2} h_{0}} .
$$

\footnotetext{
* The terminology 'local universal relation' and 'global universal relation' was defined in [2]. These refer to universal relations among quantities defined for a material element or for quantities defined for the cylinder, respectively.
} 
Since the twisting moment $T \neq 0$, the normal stress on this slanted surface can vanish if and only if (3.13) holds. By (4.1), this relation has the following form in terms of global variables,

$$
\left(\frac{R_{0}}{L_{0}}\right)^{2}=\left(\frac{R}{L}\right)^{2}\left[1-\left(\frac{R_{0}}{L_{0}}\right)^{2} \psi\right] .
$$

When (3.13) is used, (4.3) reduces to

$$
\sigma_{11}=2 \kappa \sigma_{12},
$$

which by use of (4.1) and (4.2) is equivalent to

$$
\frac{p}{T}=\frac{\psi}{\pi R_{0}^{2} L_{0}}\left(\frac{R_{0}}{R}\right)^{2}\left(\frac{L_{0}}{L}\right),
$$

a special case of (4.4).

The local homogeneous deformation of each material element of the cylinder will correspond to that discussed in Section 3, with $\sigma_{22}=N_{1}=0$, if when (4.6) holds, the pressure and twisting moment satisfy (4.8). This is true for any isotropic elastic solid, compressible or incompressible.

As a final comment, note that if $p, R$ and $L$ are held fixed, then (4.4) represents a torque-twist relation which is valid for any isotropic-elastic solid. If (4.6) is to also be met, then only $p$ and $R$ or $p$ and $L$ can be held fixed. If $p$ and $L$ are held fixed, then (4.8) becomes

$$
\frac{p}{T}=\frac{\psi}{\pi R_{0}^{2} L_{0}\left(\frac{L}{L_{0}}\right)^{3}\left[1-\left(\frac{R_{0}}{L_{0}}\right)^{2} \psi^{2}\right]}
$$

\section{Isotropic elastic solid-fluid mixtures}

Consider a mixture of an isotropic elastic solid and a fluid. The concern here is with an equilibrium state in which the fluid is uniformly dispersed throughout a homogeneously deformed solid. The general theory for such mixtures has been presented elsewhere (see for example Shi, Rajagopal and Wineman [3]). For the sake of brevity, only the relevant equations will be presented here.

As is usual in the theory of mixtures, it is assumed that each point in the current configuration of the mixture is occupied by a particle of the solid constituent and a particle of the fluid constituent. Each constituent is a continuum to which is ascribed the usual continuum variables. Let $S_{1}$ and $S_{2}$ denote the solid and fluid constituents, respectively. The motion of the mixture is referred to a fixed Cartesian coordinate system. The motions of the solid and fluid are denoted by

$$
x=\chi_{1}(X, t), \quad y=\chi_{2}(Y, t),
$$

respectively. The deformation gradient associated with the solid is given by

$$
F=\partial \chi_{1} / \partial X
$$


Let $\rho_{10}$ and $\rho_{20}$ denote the mass densities in the reference configuration of $S_{1}$ and $S_{2}$ before forming the mixture, respectively. Let $\rho_{1}$ and $\rho_{2}$ denote the mass densities of $S_{1}$ and $S_{2}$ in the mixed state, measured per unit volume of the mixture. The total mass density of the mixture, per unit volume of the mixture is

$$
\rho=\rho_{1}+\rho_{2} .
$$

The conservation of mass statement for the solid is

$$
\rho_{1} \operatorname{det} \boldsymbol{F}=\rho_{10} .
$$

It is assumed that the volume occupied by the solid and fluid before mixing equals the volume after mixing. This volume additivity requirement leads to the following constraint [4]

$$
\frac{\rho_{1}}{\rho_{10}}+\frac{\rho_{2}}{\rho_{20}}=1 .
$$

Let $\sigma_{1}$ and $\sigma_{2}$ denote the partial stress tensors associated with $S_{1}$ and $S_{2}$, respectively. The total stress for the mixture is $\sigma=\sigma_{1}+\sigma_{2}$. The equations expressing the balance of linear momenta for $S_{1}$ and $S_{2}$ are automatically satisfied in the case of the homogeneous deformation treated here. The balance of moment of momentum states that the total stress is symmetric.

Let $\boldsymbol{n}$ denote the unit outward normal vector on the boundary of the mixture. If $\boldsymbol{T}$ denotes the total traction vector on the boundary, then

$$
\boldsymbol{T}=\boldsymbol{\sigma} \boldsymbol{n} \text {. }
$$

In the development of constitutive equations for the mixture, the Helmholtz free energy per unit mass of the mixture $W$ is introduced. For a mixture of an isotropic elastic solid and a fluid, $W$ is assumed to depend on $F, \operatorname{grad} F, \rho_{2}, \operatorname{grad} \rho_{2}$, and the velocities and velocity gradients of $S_{1}$ and $S_{2}$. It has been shown in [3] that $W$ has the form

$$
W=W\left(I_{1}, I_{2}, \rho_{2}\right),
$$

where $I_{1}, I_{2}$ are the principal invariants of the Cauchy-Green tensor $\boldsymbol{B}=\boldsymbol{F} \boldsymbol{F}^{T}$. Note that by (5.4) and (5.5)

$$
I_{3}=(\operatorname{det} F)^{2}=\left(1-\frac{\rho_{2}}{\rho_{20}}\right)^{-2},
$$

and hence $I_{3}$ need not appear as an argument in (5.7).

The constitutive equation for the total stress is (cf. [3])

$$
\boldsymbol{\sigma}=-\left(p+\rho \rho_{2} \frac{\partial W}{\partial \rho_{2}}\right) \boldsymbol{I}+\rho \hat{\boldsymbol{\sigma}},
$$

where $p$ is an indeterminate scalar arising from the volume additivity constraint (5.5).

Now, let the solid constituent undergo the homogeneous deformation described in (2.1) with $\kappa \neq 0$. Furthermore, let the fluid constituent be uniformly distributed throughout the solid and in a state of rest. The configuration of the solid constituent coincides with the configuration of the mixture. Let $N_{1}$ and $L$ now denote the total normal and shear tractions, respectively, on the surface $X=X_{1}^{0}$. Since (5.9) is of the form (3.6), the universal relation (3.4) also applies to the present mixture, whatever the 
fluid content. Thus, (3.7)-(3.10) also hold. Finally suppose normal tractions vanish on $X_{1}=X_{1}^{0}$ and $X_{2}=X_{2}^{0}$, i.e. $\sigma_{22}=N_{1}=0$. Then the arguments presented in Section 3 imply that (3.13) also holds for the present mixture whatever the fluid content.

Suppose the solid constituent has been placed in a bath of fluid, and has reached a saturated state of homogeneous deformation. The equation of state relating the total tractions to the deformation has the form (Rajagopal, Wineman and Gandhi [5])

$$
\boldsymbol{\sigma}=\rho_{20}\left[W+\frac{\partial W}{\partial \rho_{2}} \frac{\rho}{I_{3}}\right] \boldsymbol{I}+\rho \hat{\boldsymbol{\sigma}} .
$$

Let the solid constituent in a deformed state described by (2.1) with $\kappa \neq 0$ be subjected to shear traction but $\sigma_{22}=N_{1}=0$, and let it be saturated with fluid. Then since (5.10) is of the form (3.6), it follows that (3.13) again holds.

Since relation (3.13) is independent of $W$, it is a 'universal relation' for any mixture of an isotropic solid and fluid, ranging from the case of a pure solid to a fully saturated solid. This relation is independent of the normal stress on $X_{3}=X_{3}^{0}$.

An immediate consequence of the above analysis is the existence of a universal relation analogous to (4.4) in the case of the extension, twisting and inflation of a thin-walled circular cylindrical mixture of a non-linearly elastic isotropic solid and fluid. It is worth noting that the relation is once again independent of the amount of the fluid in the mixture.

\section{Acknowledgement}

K.R. Rajagopal thanks the solid mechanics program of the National Science Foundation for its support.

\section{References}

[1] C. Truesdell and W. Noll, The Non-Linear Field Theories of Mechanics, Handbuch der Physik III/3 (1965).

[2] A. Wineman and M. Gandhi, On local and global universal relations in elasticity. J. Elasticity, 14, (1984), 97-102.

[3] J.J. Shi, K.R. Rajagopal and A.S. Wineman, Applications of the theory of interacting continua to the diffusion of a fluid through a non-linearly elastic media, Intl. J. Eng. Sci., 19, (1981) 871.

[4] N. Mills, Incompressible mixtures of Newtonian fluids, Intl. J. Eng. Sci, 4, (1960) 97.

[5] K.R. Rajagopal, A.S. Wineman and M. Gandhi, On boundary conditions for a certain class of problems in mixture theory, Intl. J. Eng. Sci. 24 (1986) 1453. 